\title{
Self-Assembled Peptide Nanofiber Templated One-Dimensional Gold Nanostructures Exhibiting Resistive Switching
}

\author{
Handan Acar, Rukan Genc, Mustafa Urel, Turan S. Erkal, Aykutlu Dana,* and Mustafa O. Guler* \\ Institute of Materials Science and Nanotechnology, National Nanotechnology Research Center (UNAM), Bilkent University, Ankara, \\ 06800, Turkey
}

\section{Supporting Information}

ABSTRACT: An amyloid-like peptide molecule self-assembling into one-dimensional nanofiber structure in ethanol was designed and synthesized with functional groups that can bind to gold ions. The peptide nanofibers were used as templates for nucleation and growth of one-dimensional gold nanostructures in the presence of ascorbic acid as reducing agent. We performed multistep seed-mediated synthesis of gold nanoparticles by changing peptide/gold precursor and peptide/ reducing agent ratios. Gold nanostructures with a wide range of morphologies such as smooth nanowires, noodle-like onedimensional nanostructures, and uniform aggregates of spherical nanoparticles were synthesized by use of an environmentally friendly synthesis method. Nanoscale electrical properties of gold-peptide nanofibers were investigated using atomic force microscopy. Bias dependent current (IV) measurements on thin films of gold-peptide nanofiber hybrid revealed tunneling dominated transport and resistive switching. Gold-peptide nanofiber composite nanostructures can provide insight into electrical conduction in biomolecular/inorganic composites, highlighting their potential applications in electronics and optics.

\section{INTRODUCTION}

Recent developments in controlled assembly of inorganic nanoparticles into functional macrostructures with tunable properties have provided promising results in diverse applications. ${ }^{1}$ One of the most favorable simple and costeffective methods for shape- and size-controlled assembly of nanoparticles is template-directed synthesis. ${ }^{2}$ In this approach, deposition of the inorganic material on the template can be obtained either by molecular cross-linking or by use of complementary interactions, such as electrostatic interactions, as well as layer by layer self-assembly of target ions with the help of materials acting as molecular glues (e.g., polyelectrolytes). ${ }^{3}$ Hence, control over the shape and the size of the resulting nanostructures can be achieved by geometry and surface properties of the template material. ${ }^{4,5}$ Patterns can be made up of wide variety of materials including solid inorganic materials (e.g., gold and silver) or biological materials (e.g., DNA, viruses, and polymers). ${ }^{6}$ Therefore, organization of the resulting nanoparticles and their properties achieved via template-directed synthesis are versatile. Recently, there have been increased numbers of reports on incisively designed template materials using biomimetic approaches. Tube-shaped tobacco mosaic viruses (TMV) have been studied extensively for nanoparticle synthesis due to their external surface being rich in charged amino acid residues leading binding sides. ${ }^{7}$ In another study, bacteria cellulose fibers were used for one-step preparation of gold-bacteria cellulose nanocomposites where gold was reduced and linked using poly(ethyleneimine). ${ }^{8}$ Amyloid-like peptide (ALP) nanostructures also provide quite versatile opportunities for controlled aggregation of metal nanoparticles into one-dimensional nanostructures which are of interest due to their exciting physical, mechanical, and chemical properties. $^{2,9}$ ALP nanostructures can be specifically designed to have chemically active groups offering metal binding sides on their external surface. Besides, their self-assembly into onedimensional nanostructures can occur in quite mild and diverse conditions for biomineralization. Previously, Schebeil et al. studied layer by layer electrodeposition of gold and silver on ALP nanofibers. They obtained conductive properties by peptide-nanoparticle hybrids comparable to that of solid metal wire. ${ }^{10}$ Reches et al. developed a method where assembly of silver ions into silver nanowires was driven by citrate. ${ }^{11} \mathrm{Lu}$ et al. reported nanotubes of $\mathrm{A} \beta(16-22)$, an amyloid peptide, also acting in Alzheimer disease, for gold and palladium assembly using positive charges on the nanotube surface to nucleate particle accumulation. ${ }^{12}$

Previously, we designed a self-assembling peptide, AcKFFAAK-Am, and used it for one-dimensional titania and silica nanostructure formation. ${ }^{13}$ The designed peptide molecule (Scheme 1) has amine groups acting as a hard base in the medium with affinity for hard acidic ions according to Pearson's hard soft acid base theory. ${ }^{14}$ The anionic gold complex affinity on free amine groups is a well-known strategy. ${ }^{15}$

In this work, nanofibers formed by amyloid-like peptides (ALP) were used as a template material for gold nanoparticle

Received: August 31, 2012

Revised: October 24, 2012

Published: October 30, 2012 
Scheme 1. Schematic Presentation of Gold Nanostructure Formation Using Peptide Nanofibers As Template

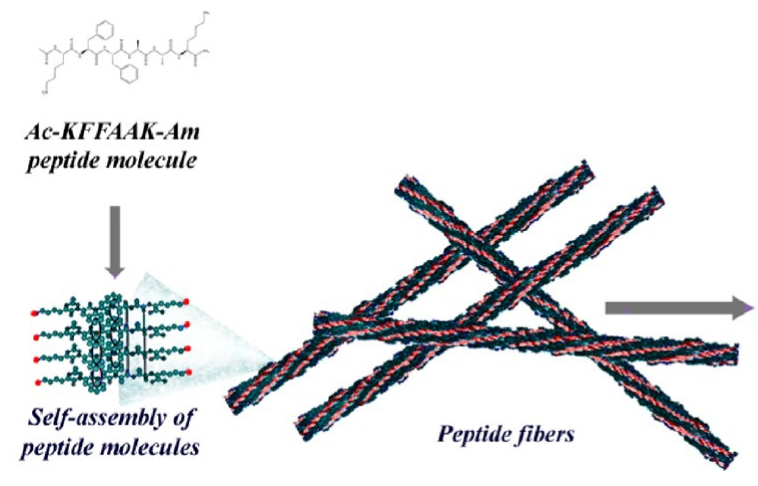

deposition exploiting the amine groups for seeding gold ions in order to drive further aurophilic interaction leading to compact nanoscale one-dimensional gold aggregates. A multistep seeding mediated growth method was adapted to template directed nanostructure synthesis where the nanocomposites can have potential use in electronic device development seeking conductive materials beyond their possible catalytic activity. ${ }^{16}$ Several parameters including time of preseeding, nanoparticle formation after addition of reducing agent, and effect of peptide concentration were studied in terms of their effect on the structural characteristics of one-dimensional gold nanostructures and template role of the peptide nanofibers. Particle assembly on the ALP nanofibers was obtained by reduction of ionic gold by ascorbic acid. Structural properties of the peptide nanofibers and assembled one-dimensional gold nanoparticles were characterized. In addition, conductivity of gold-peptide nanofiber hybrid was investigated for potential electronic applications.

\section{EXPERIMENTAL SECTION}

Peptide Synthesis. Fmoc and Boc protected amino acids, MBHA Rink Amide resin, and HBTU were purchased from NovaBiochem and ABCR. The other chemicals were purchased from Fisher, Merck, Alfa Aesar, or Aldrich and used as received. Peptide was synthesized on MBHA Rink Amide resin. Amino acid coupling reactions were performed with 2 equiv of Fmoc protected amino acid, 1.95 equiv of HBTU, and 3 equiv of DIEA for $2 \mathrm{~h}$. The Fmoc protecting group removal was performed with $20 \%$ piperidine/DMF solution for 20 min. Cleavage of the peptides from the resin was carried out with a mixture of TFA/TIS/ $\mathrm{H}_{2} \mathrm{O}$ in the ratio of 95:2.5:2.5 for $3 \mathrm{~h}$. Excess TFA was removed by rotary evaporation. The remaining peptide was triturated with ice-cold diethyl ether and the resulting white precipitate was freeze-dried. The peptide was characterized by a quadruple-timeof-flight mass spectrometry (Q-TOF MS) (SI Figure S6). The mass spectrum shows the corresponding mass of the peptide; the purity of the peptide was assessed by RP-HPLC.

Preparation of Gold Nanocomposites in Ethanol. $6.65 \times 10^{-6}$ mol peptide is mixed in $500 \mu \mathrm{L}$ of ethanol and sonicated for $5 \mathrm{~min}$ at $35^{\circ} \mathrm{C}$ for formation of clear self-supporting gel composed of peptide nanofibers. Gold was added immediately after gel preparation; $6.65 \times$ $10^{-6} \mathrm{~mol} \mathrm{KAuCl}_{4}$ (Alfa Aesar) was dissolved in $50 \mu \mathrm{L}$ of ethanol and mixed gently with a pipet tip. Samples were left on the bench at room temperature. Then, $6.65 \times 10^{-6} \mathrm{~mol} \mathrm{~L}-(+)$-ascorbic acid (Alfa Aesar) was dissolved in $10 \mu \mathrm{L}$ of water. The resulting $\mathrm{pH}$ of the solution was 2 and the solution was added directly into the gold-seeded gel.

Preparation of One-Dimensional Gold Nanocomposites via a Multistep Seed Mediated Growth Method. The multistep seed mediated growth method ${ }^{17}$ was adapted for formation of gold nanoparticles and amyloid-like peptide (ALP) nanofibers were used as template. First, $6.65 \times 10^{-6} \mathrm{~mol}$ peptide was dissolved in $500 \mu \mathrm{L}$ ethanol $(13.3 \mathrm{mM})$ and sonicated for $5 \mathrm{~min}$ at $35^{\circ} \mathrm{C}$ for formation of

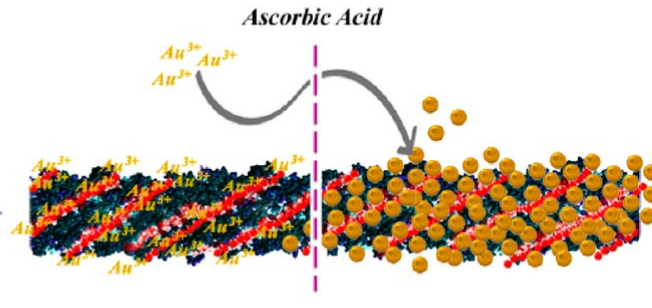

Gold nanoparticles coated peptide fibers

self-supporting gel composed of peptide nanofibers. Nuclei formation was carried out by adding $1.33 \times 10^{-6} \mathrm{~mol} \mathrm{KAuCl}_{4}$ (Alfa Aesar) dissolved in $10 \mu \mathrm{L}$ of ethanol (one-fifth of the total amount of gold) into the gel immediately after preparation and mixed gently with a pipet tip. The $\mathrm{pH}$ of the ascorbic acid solution was adjusted with $1 \mathrm{M}$ $\mathrm{NaOH}$ solution. Ten microliters of the ascorbic acid solution in water was added into $1 \mathrm{~mL}$ of preseeded gels. Solutions were aged at room temperature and samples were diluted to targeted peptide concentration by using $1.33 \times 10^{-6} \mathrm{~mol} \mathrm{KAuCl}_{4}$. Later, $10 \mu \mathrm{L}$ of ascorbic acid solution was added into the sample following a gentle mix with pipet tip. The solution was incubated for $30 \mathrm{~min}$. The seeding and reducing cycles were repeated until the desired peptide concentration was reached. The dilution factor was kept at $10 \%$ until the peptide concentration of $8.86 \mathrm{mM}$ was reached. Lower peptide concentrations were obtained with sequential dilutions of preseeded peptide gel by $50 \%$ with fresh solutions of $\mathrm{KAuCl}_{4}$ until the peptide concentration of $0.4 \mathrm{mM}$ was reached. Samples after each cycle was aged overnight and used for further characterizations.

UV-vis Absorbance. UV-vis absorbance measurements were performed on a Varian Cary 5000 UV-vis-NIR spectrophotometer. One millimeter quartz cuvettes were used for spectrophotometric analysis. Spectrophotometric analysis of gold nanoparticle formation on the preseeded peptide nanofibers with gold precursor $\left(\mathrm{KAuCl}_{4}\right.$, $13.3 \mathrm{M}$ ) reduced by changing the amount of ascorbic acid and incubation time is shown in Figure 2.

Scanning Electron Microscopy (E-SEM). SEM experiments were performed with FEI Quanta 200 FEG. A $10 \mu \mathrm{L}$ sample was dropped onto clean silicon wafer. No further coating was performed.

Transmission Electron Microscopy and Scanning Transmission Electron Microscopy. FEI Tecnai G2 F30 instrument was used for TEM analysis. Diluted samples were placed on a Lacey mesh ultrathin carbon coated copper grid. A $2 \%(\mathrm{w} / \mathrm{v})$ uranyl acetate solution was used for staining bare peptide nanofibers. Ten microliters of diluted sample solution was dropped on a grid for $1 \mathrm{~min}$. Excess was removed by pipetting. Then, 2 wt $\%$ uranyl acetate solution was placed on a parafilm sheet. The grid was put on the drop upside down for 5 min. After staining, the grids were dried in the fume hood at room temperature overnight. No staining was performed for gold nanoparticle coated peptides. Gold nanoparticles formed on the surface of peptide nanofibers prepared with $13.3 \mathrm{mM}$ peptide and an equivalent amount of $\mathrm{KAuCl} 4$ in ethanol, which was imaged after 1 week of incubation at room temperature (Figure 1).

Atomic Force Microscopy (AFM). Atomic force microscopy experiments were carried out using a commercial system; Asylum Molecular Force Probe 3D (MFP3D). Multifrequency Kelvin Probe Force Microscopy is used to measure the surface potential and capacitance. A low frequency $\left(\mathrm{f}_{\mathrm{e}}=27.5 \mathrm{kHz}\right)$ sinusoidal bias is applied to the tip during tapping mode imaging, and both harmonics (at fe corresponding to surface potential and $2 \mathrm{f}_{\mathrm{e}}$ corresponding to tipsample capacitance) are recorded. Topography was also recorded simultaneously. A DC bias was also applied to the tip superimposed with the sinusoidal bias. Standford Research Systems SR830 and SR844 lock-in amplifiers were used to measure the time harmonics of 


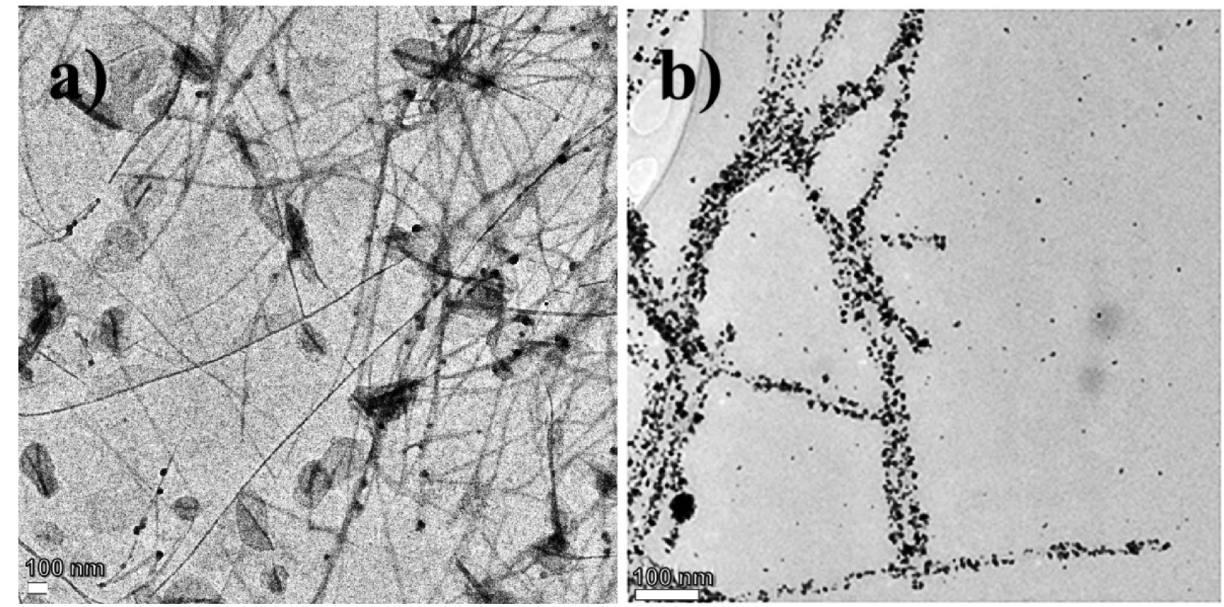

Figure 1. Representative TEM images of (a) peptide nanofibers stained with uranyl acetate and (b) gold nanoparticles formed on the surface of peptide nanofibers.

the electrostatic force. The samples were scanned at a rate between 0.15 and $0.5 \mathrm{~Hz}$. Commercial $\mathrm{Cr} / \mathrm{Pt}$ coated cantilevers were used, with resonance frequencies about $70 \mathrm{kHz}$ and spring constants of $2-4 \mathrm{~N} /$ $\mathrm{m}$. Tapping drive frequencies were selected to favor repulsive mode imaging. Biases to the contacts on the surface were applied by using Keithley 2400.

Preparation of Contacts for AFM and Probe Station Electrical Measurements. A p-Si $\langle 100\rangle 4$ in. wafer was coated with $1-\mu \mathrm{m}$-thick $\mathrm{SiNx}$ as an isolation dielectric layer. Contacts with different gap lengths between 2 and $50 \mu \mathrm{m}$ were formed by photolithography and subsequent metallization (sputtering of $35 \mathrm{~nm}$ of $\mathrm{Au} / \mathrm{Pd}$ ). The peptide and gold nanocomposites were drop-cast onto the substrates after possible dilution.

\section{RESULTS AND DISCUSSION}

Affinity of the gold to form complex with free amine groups is a well-known strategy. ${ }^{15}$ Herein, an amyloid-like peptide (ALP) was designed to form nanofibers of micrometers in length with amine groups on their exterior surface (Scheme 1). The amine groups located on the periphery of the peptide nanofibers can bind to $\mathrm{AuCl}_{4}^{-} / \mathrm{AuCl}_{2}{ }^{-}$ions by electrostatic interactions. ${ }^{18,19}$ Electrostatic interactions between $\mathrm{AuCl}_{4}{ }^{-} / \mathrm{AuCl}_{2}{ }^{-}$ions and protonated amine groups cause gold adsorption on the outer surface of the peptide nanofibers. ${ }^{20}$ Moreover, the ALP nanofibers form self-supporting gels in ethanol (Figure 1a). ${ }^{21,22}$

The structural properties and corresponding surface plasmon resonance (SPR) characteristics of the nanostructures were examined by TEM imaging and UV spectrophotometer to study the appropriate ratio of the peptide and $\mathrm{KAuCl}_{4}$. After one week of incubation at room temperature, particle formation around the peptide nanofibers was observed. However, using higher molar ratios of $\mathrm{KAuCl}_{4}$ and peptide (2 and 3 molar ratios) leads to a dramatic increase in the number of free randomly distributed gold nanoparticles (SI Figure S1 and Figure $1 b$ ). Nanoparticles formed at the surface of the peptide nanofibers did not show a characteristic plasmon peak at 550 $\mathrm{nm}$ (SI Figure S2); however, a strong peak at $320 \mathrm{~nm}$ was observed due to ionic $\mathrm{AuCl}_{4}{ }^{-}$in solution. ${ }^{23}$ The absence of a size-dependent localized plasmon resonance effect might be because of the small particle size below the critical value or hindrance of SPR feature as a result of the closer gap between the particles located through the peptide nanofibers. ${ }^{24}$

Although nanoparticle alignment around the peptide nanofibers was observed with ethanol, the reduction capacity of ethanol was not sufficient to obtain full arrangement of nanoparticles in one dimension. Ascorbic acid was used as a reducing agent in preparation of one-dimensional gold nanostructures by facilitating the coordination of metal precursor at postseeding stages. ${ }^{25-27}$ Ascorbic acid was added directly in gold precursor solution or added in a peptide nanofiber system, which was preseeded overnight with gold salt. In the first approach, neither a color change nor nanoparticle formation was observed, whereas micrometer-sized randomly distributed aggregates were found in the SEM images (SI Figure S3). On the other hand, a change in a couple of minutes from yellowish to transparent and later to dark brown was observed when ascorbic acid was added to preseeded peptide nanofiber solution. UV-vis absorbance spectrophotometer measurements and electron microscopy techniques were used to analyze reaction kinetics of ascorbic acid as a reducing agent. Consumption of gold ions can be tracked by change in characteristic peak at $320 \mathrm{~nm}$, and it was monitored by changing ascorbic acid concentration over time. At increased ascorbic acid concentrations and constant $\mathrm{KAuCl}_{4}$ concentration, the peak at $320 \mathrm{~nm}$ showed fast decrease until 1:1 molar ratio of ascorbic acid to gold (Figure 2a). The same samples were analyzed by SEM and we observed that the number of free particles was increased with increasing amount of ascorbic acid. The UV-vis spectrum of the same sample showed a broad peak with a maximum around $550 \mathrm{~nm}$ (Figure 2a). As shown in the inset of Figure 2a, peak maxima at 320 and $550 \mathrm{~nm}$ have inverse relationship. To obtain maximum conversion of $\mathrm{Au}^{3+}$ to $\mathrm{Au}^{0}$ and minimize the formation of free nanoparticles, ascorbic acid concentration was determined by the data point where two lines intercepts revealing 1:1 molar ratio of ascorbic acid to gold. This ratio was taken as the optimum ratio for this study.

After determining optimum ascorbic acid concentration, time dependence of the particle assembly on peptide fibers was analyzed by adding ascorbic acid solution ( $13.3 \mathrm{mM}$ ) into preseeded peptide nanofibers with $\mathrm{KAuCl}_{4}(13.3 \mathrm{mM})$, and the sample was monitored for over a week. Thirty minute after ascorbic acid addition, the peak at $320 \mathrm{~nm}$ decreased to about its one-fifth, and no further change was observed (Figure $2 b$ ). On the other hand, the peak at $550 \mathrm{~nm}$ started to dominate after $30 \mathrm{~min}$ displaying an increased amount of gold nanoparticles (Figure 2b, inset) in the surrounding medium, which was also observed in SEM imaging (SI Figure S3). To 

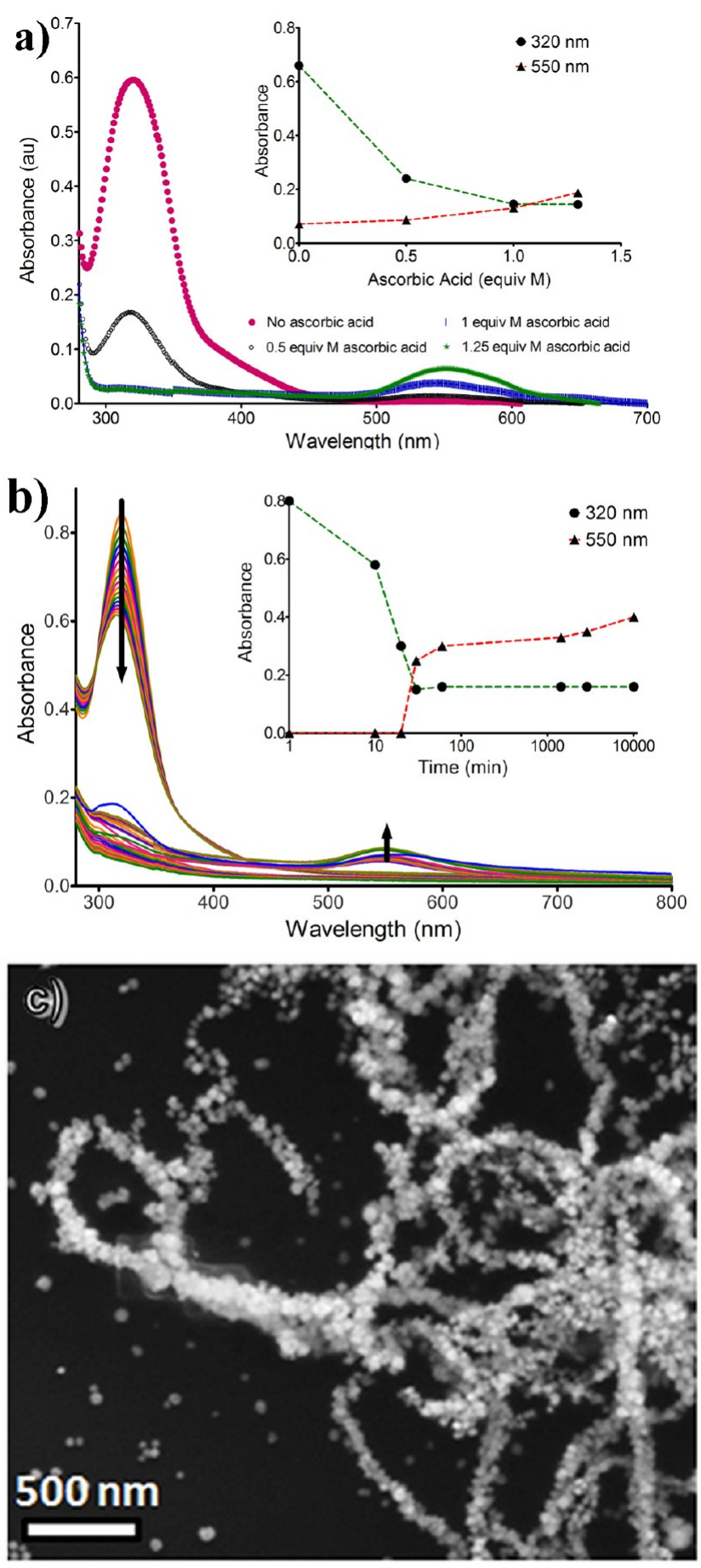

Figure 2. (a) Spectrophotometric analysis of gold nanoparticle formation on the peptide nanofibers at changing concentration of ascorbic acid at $60 \mathrm{~min}$ of incubation (Inset shows change in absorbance at 320 and $550 \mathrm{~nm}$ depending on the ascorbic acid concentration). (b) As time passes the absorption at $320 \mathrm{~nm}$ decreases while $550 \mathrm{~nm}$ increases at $13.3 \mathrm{mM}$ ascorbic acid concentration (Inset shows relationship between peak maxima at 320 and $550 \mathrm{~nm}$ ). (c) SEM image of gold nanoparticles formed on the peptide nanofiber template at optimized incubation time and reducing agent concentration.

figure out optimum incubation time, the lowest value possible for both peaks at 320 and $550 \mathrm{~nm}$ was taken. High-aspect-ratio gold nanostructures (Figure 2c) were obtained at this lowest point with fewer unbound nanoparticles compared to the ones synthesized by incubating longer (SI Figure S3). Therefore, we conclude that the optimum time for ascorbic acid to reduce the gold residues on the template surface with the formation of fewer free nanoparticles is $30 \mathrm{~min}$.

After optimization of the initial conditions, a multistep seedmediated growth method inspired from Jana et al. ${ }^{28}$ was modified in order to obtain nanowires with higher organization by tuning concentration of peptide solutions and gel density, which is related to the peptide concentration. ${ }^{29}$ Mass transfer of the reactants is affected by the density of the peptide nanofiber gel. At higher peptide concentrations, there are more peptide nanofibers in the medium, and a dense network of peptide nanofibers results in less porous system. Therefore, slower diffusion of reagents is expected in the denser network preventing uniform distribution of the reagents (SI Scheme S1) ${ }^{30,31}$ Decreasing peptide concentration will lead to decrease in the amount of peptide nanofibers in the medium. Therefore, increased porosity eventually facilitates molecular mobility resulting in a more uniform distribution of reaction species in the medium and minimizes random gold reduction. In order to study the effect of peptide concentration on the final nanostructure, gold nanowires were formed at peptide concentrations from 11.9 to $0.4 \mathrm{mM}$ and were monitored by transmission electron microscopy (SI Figure S4 and S5). After addition of the first gold precursor, the $\mathrm{AuCl}_{4}{ }^{-} / \mathrm{AuCl}_{2}{ }^{-}$ions started to bind on the protonated amine groups, and with the help of the first ascorbic acid addition, gold seeds (about 1-2 $\mathrm{nm}$ diameter of nanoparticles) were observed (SI Figure S4). Decreasing peptide concentration from 11.9 to $8.9 \mathrm{mM}$ (SI Figure S5) revealed a transition from nodule-like nanowires to nanowires of compact arrangement of nanoparticles due to particle merging on the seeds formed in the previous stage. In Figure 3a, gold nanowires were clearly observed in samples prepared by $6.6 \mathrm{mM}$ peptide concentration where packing of nanoparticles in diameter of $3.50 \pm 0.71 \mathrm{~nm}$ is prominent. The XRD pattern of these nanowires revealed a strong signal at $38^{\circ}$, which corresponds to (111) plane of Au crystal (Figure 3b) and indicates that the (111) plane is dominant and is parallel to the support surface. ${ }^{32}$ This result is consistent with STEM images (Figure $3 \mathrm{a}$ and SI Figure S5). The calculated intensity of $(200) /(111)$ of 0.165 was lower than that of the characteristic value of nanowires (0.249), which was defined in the standard file JCPDS. ${ }^{32}$ No surface plasmon peak was observed. However, a broad UV-vis absorbance peak (Figure 3c) was observed between 500 and $700 \mathrm{~nm}$. A similarly broad signal for nanowires composed of nanoparticles arranged on a template was reported previously. ${ }^{8}$ The nanofibers are coated with smalldiameter gold nanoparticles, and the electromagnetic properties of such closely separated nanoparticle arrays significantly differ from those of monolithic gold nanorods due to the presence of gaps between the nanoparticles. The $\mathrm{Au}$ nanoparticles were observed to have diameters below $15 \mathrm{~nm}$, and it is wellestablished both theoretically and experimentally that $\mathrm{Au}$ nanoparticles and rods begin to exhibit SPR peaks when the characteristic size exceeds ca. $30 \mathrm{~nm}$. Therefore, it is reasonable not to expect transverse resonances due to few nanometers of $\mathrm{Au}$ nanoparticles configured around a typical fiber diameter of less than $10 \mathrm{~nm}$. The longitudinal resonances are also absent due to the lack of a continuous gold layer as well as infinite nanofiber length, which would be necessary to observe welldefined resonances even in the presence of a continuous gold film. ${ }^{33,34}$ The results are consistent with the TEM images showing densely packed gold nanoparticle alignment on the 

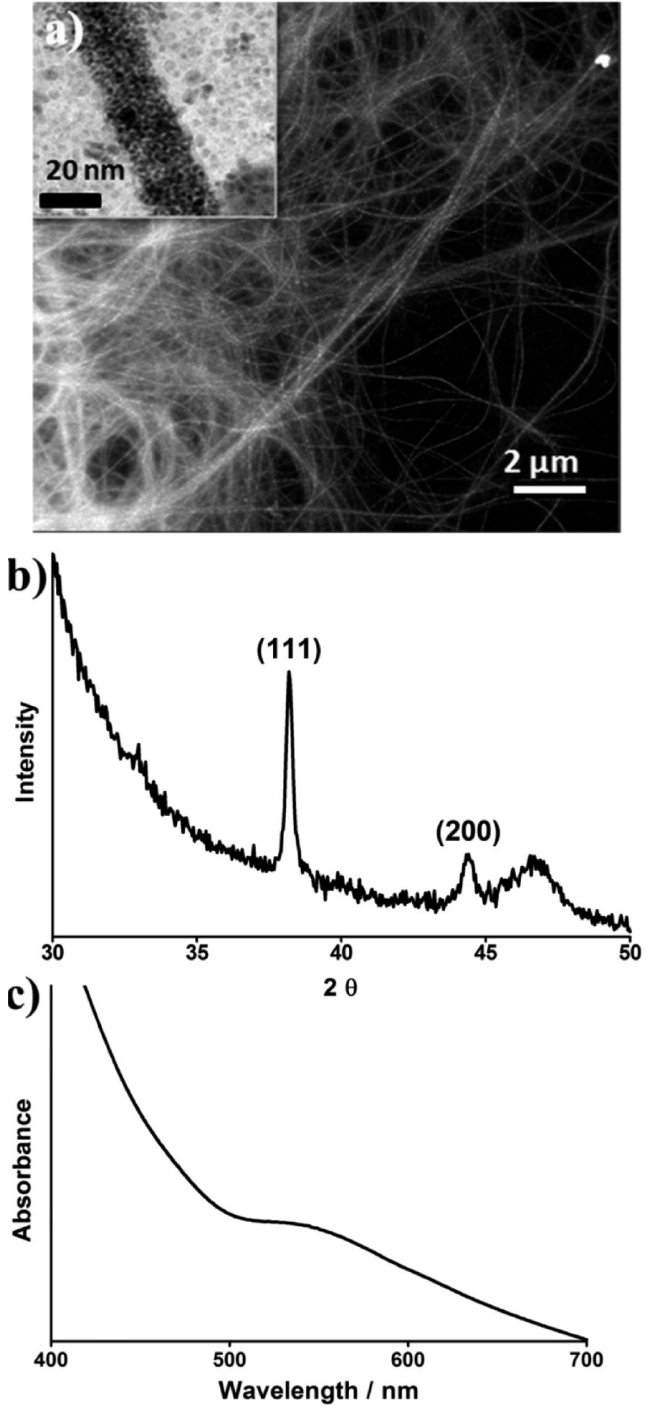

Figure 3. (a) STEM image of gold nanowires synthesized using multistep seed-directed methodology, (b) XRD pattern, and (c) absorbance spectrum of the nanostructures.

ALP nanofiber surface. On the other hand, at peptide concentration below $6.6 \mathrm{mM}$, the template effect was disturbed and nanoparticle agglomeration was observed (SI Figure S5). $\mathrm{XRD}$ patterns are weak to calculate in this sample. As predicted, multistep seed-mediated methodology favored controlled formation of high-aspect-ratio gold nanostructures composed of smaller gold nanoparticles with higher degree of packing (Figure 3a) compared to the bulk methodology where ascorbic acid was added directly to the peptide gel (Figure 2c).

The reduction capacity of ascorbic acid is dependent on the $\mathrm{pH}$ of the ascorbic acid solution. ${ }^{35-37}$ At alkaline $\mathrm{pH}$, ascorbate is a stronger reducing agent than its acid form. ${ }^{38,39}$ Increased $\mathrm{NaOH}$ amount in the ascorbic acid solution led to formation of uniform nanoparticles with diameter of about $20 \mathrm{~nm}$. However, they were mostly located randomly on the clusters of peptide nanofibers with no template effect. Instant color change from pale yellow to blackish purple is also an indication of the aggregate formation. Fast reduction due to increased ascorbate concentration resulted in uncontrolled growth over the template (SI Figure S5). Required time for ascorbic acid to reduce the ionic gold to form one-dimensional nanocomposites in the presence of peptide nanofibers was $30 \mathrm{~min}$. However, longer time for reduction between dilutions can initiate formation of highly crystalline one-dimensional structures formed by gold nanoparticles. As shown in Figure 4a, peptide concentration of $6.6 \mathrm{mM}$ revealed nanowires of fully merged gold nanoparticles with a fairly narrow and dominant $(2 \Theta)$ at $38^{\circ}$ corresponding to (111) crystal plane.
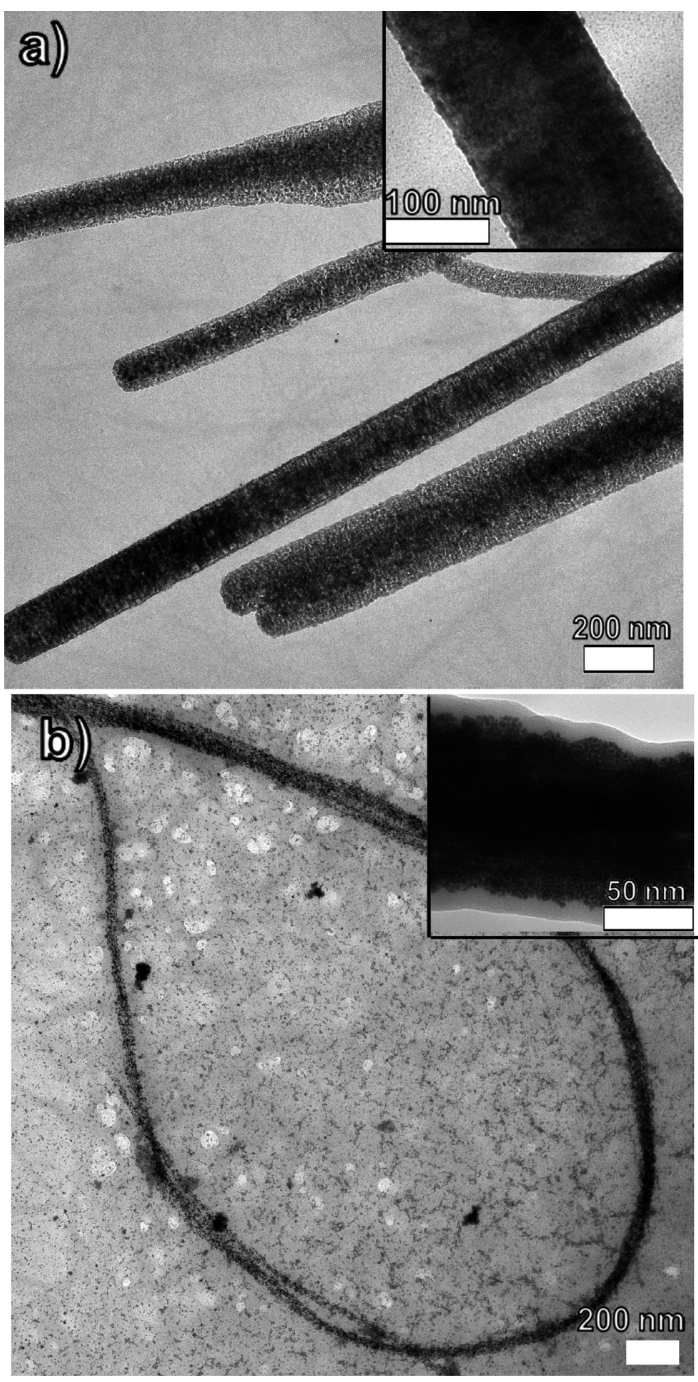

Figure 4. TEM images of gold nanostructures formed after 1 day of aging. (a) Gold nanowires obtained in the presence of $6.6 \mathrm{mM}$ peptide and (b) peptide surrounded nanowires assembled in the presence of $1.19 \times 10^{-2} \mathrm{M}$ peptide.

However, when peptide concentration was increased to 11 $\mathrm{mM}$, we observed $\sim 5 \mathrm{~nm}$ gold nanoparticles covered by a thick peptide layer (Figure 4b). As illustrated in SI Scheme S2, the formation of the string bag-like appearance was due to high peptide concentration. Incubating longer did not initiate higher packing at this concentration, but it promoted binding of free peptide molecules to gold nanoparticles similar to a capping agent and resulted in a new layer for the nuclei formation at the following seeding steps. This stepwise accumulation was completed with the formation of cable-like packaging of the final structure with the peptide layer. These results demonstrated that, by simply aging the samples for longer times between the dilutions, structural properties of one-dimensional 

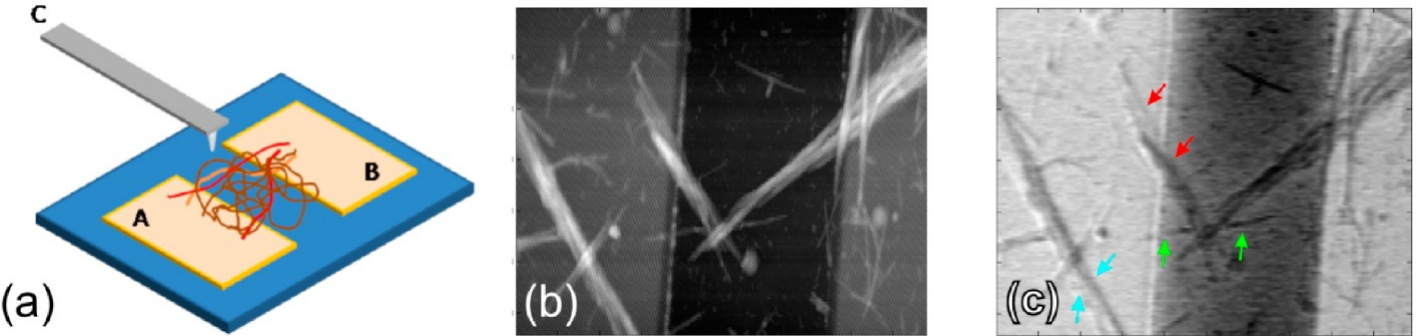

Figure 5. (a) Dilute gold-peptide nanofiber network is drop-cast on a silicon wafer with a 1- $\mu$ m-thick oxide barrier. A voltage can be applied to the cantilever (denoted by C) or to any of the AuPd contacts (denoted by A and B). (b) Simultaneous measurement of topography and (c) surface potential show the low and nonuniform conductivity of the nanofibers. Several arrows are used to denote changes in surface potential along individual nanofibers or nanofibers in contact, when a bias of $V_{\mathrm{AB}}=0.5 \mathrm{~V}$ is applied. In contrast, AuPd contacts show uniform surface potential distribution.
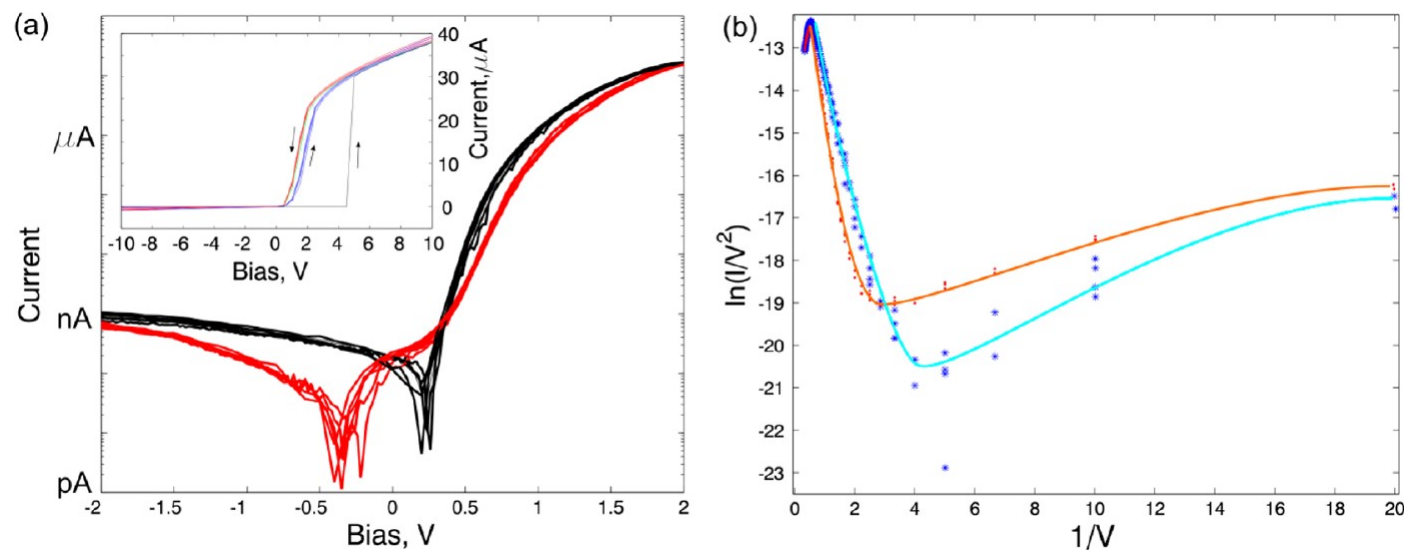

Figure 6. (a) Cyclic current versus bias measurements reveal asymmetry in the conductance as well as hysteresis. Initially, the samples have typically high-resistance, and switching to a high-conductivity state occurs around $4.5 \mathrm{~V}$ bias (see inset). Several cycles are superimposed to show the extent of the repeatability of the measurements. (b) The current is attributed primarily to tunneling as seen in the Fowler-Nordheim presentation, when $\ln (I /$ $V^{2}$ ) is plotted against $1 / V$ for increasing (red) and decreasing (blue) bias sweeps. Solid lines are guides for the eye. The transition voltage from direct to Fowler-Nordheim tunneling is a function of tunneling gap and effective tunnel barrier height, and is seen to be modulated during cyclic voltage sweeps.

gold nanocomposites can easily be tailored depending on the application.

The presence of closely packed gold nanoparticles on the peptide nanofibers, separated by peptides or thin vacuum gaps, suggests that conductivity through dry film networks of such nanofibers can be considered essentially to be through electron tunneling in a series of molecular-break-junctions. ${ }^{40}$ Conductance through random nanowire networks has attracted significant attention due to potential applications as semitransparent contacts or flexible/stretchable conductors and has been studied both theoretically and experimentally. ${ }^{41-44}$ Considering their structural similarity, the conductivity mechanisms of gold-decorated peptide nanofiber networks are expected to be similar to those of nanowire networks if the gold encapsulation can be considered a continuous coating. If the gold nanoparticle coating is discontinuous (gold nanoparticles are separated by peptides or vacuum), tunneling is expected to dominate the conduction through individual nanofibers, and overall conductivity of the network is expected to be described by a more complex model involving tunneling dominated transport within and between nanofibers. Nanoscale electrical properties of individual fibers were investigated using atomic force microscopy. Gold-peptide nanofiber composite was dropcast onto insulating substrates with AuPd contacts separated by a distance of $10 \mu \mathrm{m}$. No tunneling current could be observed between the nanofibers and contacts using moderate bias voltages below $5 \mathrm{~V}$; current levels were below the $10 \mathrm{pA} \mathrm{rms}$ noise level of the measurement system. Although the DC current can be low, quasi-static charging can allow induction of significant changes in the surface potentials of nanostructures. In order to observe changes in the surface potentials of individual fibers under bias, multifrequency Kelvin probe microscopy was used to simultaneously measure topography and surface potential, as shown in Figure $5 .{ }^{45}$ Bias is applied between the contacts (contacts $\mathrm{A}$ and $\mathrm{B}$ in Figure 5a), and a secondary bias is applied to the cantilever (contact $\mathrm{C}$ in Figure $5 a)$. We observed that locations with different potentials exist along a nanofiber or nanofibers that are in contact with each other (Figure 5b,c). This suggests that gold nanoparticle decorated peptide nanofibers are individually near the percolation threshold, with occasional gaps between gold nanoparticles.

Current-voltage $(I-V)$ characteristics of dilute gels of gold decorated peptide networks were measured through contacts A and B in Figure 5a. Typically, samples revealed high resistance, as would be expected from the low conductivity of individual nanofibers observed by AFM study. Occasionally, a high conductivity path emerges after which resistive switching behavior is observed to be repeatable (Figure 6a). Transition voltage spectroscopy (TVS) has been used to study conductance properties of molecular-break junctions. ${ }^{46,47}$ In TVS, the Fowler-Nordheim presentation of the $I-V$ data is 
used by plotting $\ln \left(I / V^{2}\right)$ against $1 / V$. In such a plot, typically an asymmetric dip is observed for a tunnel junction, which is confirmed to be the case for the Au-decorated nanofiber samples (Figure $6 \mathrm{~b}$ ). The transition voltage from direct to Fowler-Nordheim tunneling, $V_{\mathrm{m}} \approx\left(2 \hbar / \mathrm{em}^{1 / 2}\right)\left((2 \varphi)^{1 / 2} / d\right)$, is a function of tunneling gap $d$ and effective tunnel barrier height $\varphi$, where $e$ and $m$ are the electronic charge and mass. $V_{\mathrm{T}}$ was observed to be modulated during cyclic voltage sweeps, which may indicate a change in the tunnel gap $d$ or a modulation of the barrier height $\varphi$. Although it is difficult to determine the dominant mechanism leading to resistive switching without ambiguity, we speculate that electrochemical changes could be important. Due to applied bias, ions in the residual water may intercalate within the peptide nanofibers, causing swelling and contraction. Small changes in the fiber structure may modulate the effective tunnel gap and result in the observed hysteresis of the $I-V$ curve.

Measurements performed on thin films of Au-decorated and bare peptide nanofibers revealed a pronounced difference in the overall conductivity of the nanofiber network. Thicker films (as revealed by atomic force microscopy) of bare nanofibers exhibit current levels at picoampere level, while Au-nanoparticledecorated nanofibers exhibit current levels at microampere level at similar biases. These observations suggest that the Audecorated nanofiber network possesses a much higher conductivity compared to bare nanofiber networks. Atomic force microscopy based current measurements on even smaller numbers of bare nanofibers showed no detectable conductivity (with current levels below $1 \mathrm{pA}$ for -10 to $10 \mathrm{~V}$ bias range; data not shown) on bundles of few nanofibers. In addition, an increase in ambient humidity was observed to increase the current levels in both bare and Au-decorated nanofiber networks (data not shown). These observations suggest that the measured conductivity (with current levels of up to 100 $\mathrm{pA}$ ) in thin film networks of bare nanofibers may be solely due to the small ionic conductivity arising from the presence of residual ions and condensed water on the films. Further elucidation of the role of ionic conductivity requires a detailed study of the effect of humidity on conductivity of films and is left beyond the scope of this study. However, more than 1000fold increase in the conductivity of Au-decorated nanofibers along with reasonable agreement of the current-voltage dependence on the theoretical predictions point to a conduction mechanism dominated by tunneling between the $\mathrm{Au}$ nanoparticles on the nanofibers (SI Figure S9).

\section{CONCLUSIONS}

In conclusion, self-assembled amyloid-like peptide nanofibers were used as template material for one-dimensional growth of gold nanowires. Functional groups on the peptide nanofiber surface acting as binding moieties were used to initiate the seed-mediated growth of gold nanoparticles. The effect of peptide, gold precursor and ascorbic acid concentrations on morphology of the final nanostructures were studied. A multistep seeding mediated growth methodology for template-directed synthesis of one-dimensional gold nanostructure was achieved. Nanowires, noodle-like one-dimensional gold nanostructures, and homogeneous spherical gold nanoparticles were obtained in a controlled manner with the help of peptide nanofibers. The nanowires are highly conductive due to presence of a large number of gaps between gold domains, and dry films of peptide nanofibers showed tunnelingdominated conductance as well as resistive switching.
Transition voltage exhibited hysteresis, which is due to the presence of electrochemical effects and related modulation of the effective tunneling gap and barrier height. This strategy can be extended to synthesis of a wide range of metal and metal oxide nanoparticles depending on the application. For example, ultrathin and long metal-decorated nanofibers can, in principle, find use in applications where an optically transparent, low hazing, and conductive coating is needed, such as in flexible displays, touch-interface devices, and solar cells. The production strategy offers multiple parameters which would allow tuning of optical and electrical properties.

\section{ASSOCIATED CONTENT}

\section{Supporting Information}

Experimental details and additional micrographs. This material is available free of charge via the Internet at http://pubs.acs.org.

\section{AUTHOR INFORMATION}

\section{Corresponding Author}

*Telephone: +90 (312) 290 3552. Fax: +90 (312) 266 4365. Email: moguler@unam.bilkent.edu.tr (M.O.G.) and aykutlu@ unam.bilkent.edu.tr (A.D.).

\section{Notes}

The authors declare no competing financial interest.

\section{ACKNOWLEDGMENTS}

We thank M. Guler for help in TEM and STEM imaging. This work was supported by the Scientific and Technological Research Council of Turkey (TUBITAK) grant number 109T603, FP7 Marie Curie IRG Grant, and COMSTECHTWAS grant. M.O.G. acknowledges support from the Turkish Academy of Sciences Distinguished Young Scientist Award (TUBA-GEBIP). R.G. is supported by TUBITAK-BIDEB 2218 postdoctoral research fellowship.

\section{REFERENCES}

(1) Eustis, S.; el-Sayed, M. A. Why gold nanoparticles are more precious than pretty gold: noble metal surface plasmon resonance and its enhancement of the radiative and nonradiative properties of nanocrystals of different shapes. Chem. Soc. Rev. 2006, 35, 209-17.

(2) Xia, Y.; Yang, P.; Sun, Y.; Wu, Y.; Mayers, B.; Gates, B.; Yin, Y.; Kim, F.; Yan, H. One-dimensional nanostructures: synthesis, characterization, and applications. Adv. Mater. 2003, 15, 353-389.

(3) Lamm, M. S.; Sharma, N.; Rajagopal, K.; Beyer, F. L.; Schneider, J. P.; Pochan, D. J. Laterally spaced linear nanoparticle arrays templated by laminated $\beta$-sheet fibrils. Adv. Mater. 2008, 20, 447-451.

(4) Correa-Duarte, M. A.; Liz-Marzán, L. M. Carbon nanotubes as templates for one-dimensional nanoparticle assemblies. J. Mater. Chem. 2006, 16, 22-25.

(5) Yuwono, V. M.; Hartgerink, J. D. Peptide amphiphile nanofibers template and catalyze silica nanotube formation. Langmuir 2007, 23, 5033-8.

(6) Mirkin, C. A.; Letsinger, R. L.; Mucic, R. C.; Storhoff, J. J. A DNA-based method for rationally assembling nanoparticles into macroscopic materials. Nature 1996, 382, 607-9.

(7) Dujardin, E.; Peet, C.; Stubbs, G.; Culver, J. N.; Mann, S. Organization of Metallic Nanoparticles Using Tobacco Mosaic Virus Templates. Nano Lett. 2003, 3, 413-417.

(8) Zhang, T.; Wang, W.; Zhang, D.; Zhang, X.; Ma, Y.; Zhou, Y.; Qi, L. Biotemplated Synthesis of Gold Nanoparticle-Bacteria Cellulose Nanofiber Nanocomposites and Their Application in Biosensing. Adv. Funct. Mater. 2010, 20, 1152-1160.

(9) Toksoz, S.; Acar, H.; Guler, M. O. Self-assembled onedimensional soft nanostructures. Soft Matter 2010, 6, 5839-5839. 
(10) Scheibel, T.; Parthasarathy, R.; Sawicki, G.; Lin, X.-M.; Jaeger, H.; Lindquist, S. L. Conducting nanowires built by controlled selfassembly of amyloid fibers and selective metal deposition. Proc. Natl. Acad. Sci. U.S.A. 2003, 100, 4527-32.

(11) Reches, M.; Gazit, E. Casting metal nanowires within discrete self-assembled peptide nanotubes. Science 2003, 300, 625-7.

(12) Lu, K.; Conticello, V. P.; Lynn, D. G. Templating Colloidal Metal Nanoparticle Assemblies: Use of the A $\beta$ Amyloid Peptide Nanotube. Mater. Res. Soc. Symp. Proc. 2004, 826E, V1.6.

(13) Acar, H.; Garifullin, R; Guler, M. O. Self-assembled templatedirected synthesis of one-dimensional silica and titania nanostructures. Langmuir 2011, 27, 1079-84.

(14) Pearson, R. G. Hard and soft acids and bases, HSAB, part 1: Fundamental principles. J. Chem. Educ. 1968, 45, 581-581.

(15) Mack, C.; Wilhelmi, B.; Duncan, J. R.; Burgess, J. E. Biosorption of precious metals. Biotechnol. Adv. 2007, 25, 264-71.

(16) Wilms, M.; Conrad, J.; Vasilev, K.; Kreiter, M.; Wegner, G. Manipulation and conductivity measurements of gold nanowires. Appl. Surf. Sci. 2004, 238, 490-494.

(17) Gole, A.; Murphy, C. J. Seed-Mediated Synthesis of Gold Nanorods: Role of the Size and Nature of the Seed. Chem. Mater. 2004, 16, 3633-3640.

(18) Chen, W.; Deng, H.-H.; Hong, L.; Wu, Z.-Q.; Wang, S.; Liu, A.L.; Lin, X.-H.; Xia, X.-H. Bare gold nanoparticles as facile and sensitive colorimetric probe for melamine detection. Analyst 2012, 137, 53825386.

(19) Joshi, H.; Shirude, P. S.; Bansal, V.; Ganesh, K. N.; Sastry, M. Isothermal Titration Calorimetry Studies on the Binding of Amino Acids to Gold Nanoparticles. J. Phys. Chem. B 2004, 108, 1153511540 .

(20) Lotfi Zadeh Zhad, H. R.; Aboufazeli, F.; Sadeghi, O.; Amani, V.; Najafi, E.; Tavassoli, N. Tris(2-Aminoethyl)Amine-Functionalized Fe3O4Magnetic Nanoparticles as a Selective Sorbent for Separation of Silver and Gold Ions in Different pHs. J. Chem. 2013, 2013, 7.

(21) Ayyappan, S.; Gopalan, R. S.; Subbanna, G. N.; Rao, C. N. R. Nanoparticles of $\mathrm{Ag}, \mathrm{Au}, \mathrm{Pd}$, and $\mathrm{Cu}$ produced by alcohol reduction of the salts. J. Mater. Res. 1997, 12, 398-401.

(22) Liao, J. Linear aggregation of gold nanoparticles in ethanol. Colloids Surf., A 2003, 223, 177-183.

(23) Tanahashi, I.; Tohda, T. Photoinduced Formation of Small Gold Particles in Silica Gels. J. Am. Ceram. Soc. 2005, 79, 796-798.

(24) Lin, S.; Li, M.; Dujardin, E.; Girard, C.; Mann, S. OneDimensional Plasmon Coupling by Facile Self-Assembly of Gold Nanoparticles into Branched Chain Networks. Adv. Mater. 2005, 17, 2553-2559.

(25) Gray, H. B.; Ballhausen, C. J. A Molecular Orbital Theory for Square Planar Metal Complexes. J. Am. Chem. Soc. 1963, 85, 260-265.

(26) Jana, N. R.; Gearheart, L.; Murphy, C. J. Evidence for SeedMediated Nucleation in the Chemical Reduction of Gold Salts to Gold Nanoparticles. Chem. Mater. 2001, 13, 2313-2322.

(27) Jana, N. R.; Gearheart, L.; Murphy, C. J. Wet Chemical Synthesis of High Aspect Ratio Cylindrical Gold Nanorods. J. Phys. Chem. B 2001, 105, 4065-4067.

(28) Jana, N. R.; Gearheart, L.; Murphy, C. J. Seeding Growth for Size Control of 5-40 nm Diameter Gold Nanoparticles. Langmuir 2001, 17, 6782-6786.

(29) Kaufman, L. J.; Brangwynne, C. P.; Kasza, K. E.; Filippidi, E.; Gordon, V. D.; Deisboeck, T. S.; Weitz, D. A. Glioma expansion in collagen I matrices: analyzing collagen concentration-dependent growth and motility patterns. Biophys. J. 2005, 89, 635-50.

(30) Branco, M. C.; Pochan, D. J.; Wagner, N. J.; Schneider, J. P. Macromolecular diffusion and release from self-assembled beta-hairpin peptide hydrogels. Biomaterials 2009, 30, 1339-1347.

(31) Yang, Y.-L.; Leone, L. M.; Kaufman, L. J. Elastic moduli of collagen gels can be predicted from two-dimensional confocal microscopy. Biophys. J. 2009, 97, 2051-60.

(32) Sinha, A. K.; Basu, M.; Sarkar, S.; Pradhan, M.; Pal, T. Electrostatic field force directed gold nanowires from anion exchange resin. Langmuir 2010, 26, 17419-26.
(33) Bromley, K. M.; Patil, A. J.; Perriman, A. W.; Stubbs, G.; Mann, $S$. Preparation of high quality nanowires by tobacco mosaic virus templating of gold nanoparticles. J. Mater. Chem. 2008, 18, 47964801.

(34) Zhang, G.; Keita, B.; Biboum, R. N.; Miserque, F.; Berthet, P.; Dolbecq, A.; Mialane, P.; Catala, L.; Nadjo, L. Synthesis of various crystalline gold nanostructures in water: The polyoxometalate [small beta]-[H4PMo12O40]3- as the reducing and stabilizing agent. J. Mater. Chem. 2009, 19, 8639-8644.

(35) Borsook, H.; Davenport, H. W.; Jeffreys, C. E. P.; Warner, R. C. Am. Soc. Biol. Chem. 1937, 117, 237-279.

(36) Zhong, Z.; Patskovskyy, S.; Bouvrette, P.; Luong, J. H. T.; Gedanken, A. The Surface Chemistry of Au Colloids and Their Interactions with Functional Amino Acids. J. Phys. Chem. B 2004, 108, 4046-4052.

(37) Borsook, H.; Keighley, G. Oxidation-Reduction Potential of Ascorbic Acid (Vitamin C). Proc. Natl. Acad. Sci. U.S.A. 1933, 19, 8758 .

(38) Qin, Y.; Ji, X.; Jing, J.; Liu, H.; Wu, H.; Yang, W. Size control over spherical silver nanoparticles by ascorbic acid reduction. Colloids Surf., A 2010, 372, 172-176.

(39) Huang, C.-C.; Yang, Z.; Chang, H.-T. Synthesis of dumbbellshaped $\mathrm{Au}-\mathrm{Ag}$ core-shell nanorods by seed-mediated growth under alkaline conditions. Langmuir 2004, 20, 6089-92.

(40) Reed, M. A.; Zhou, C.; Muller, C. J.; Burgin, T. P.; Tour, J. M. Conductance of a Molecular Junction. Science 1997, 278, 252-254.

(41) Lee, P.; Lee, J.; Lee, H.; Yeo, J.; Hong, S.; Nam, K. H.; Lee, D.; Lee, S. S.; Ko, S. H. Highly Stretchable and Highly Conductive Metal Electrode by Very Long Metal Nanowire Percolation Network. Adv. Mater. 2012, 24, 3326-3332.

(42) White, S. I.; Mutiso, R. M.; Vora, P. M.; Jahnke, D.; Hsu, S.; Kikkawa, J. M.; Li, J.; Fischer, J. E.; Winey, K. I. Electrical Percolation Behavior in Silver Nanowire-Polystyrene Composites: Simulation and Experiment. Adv. Funct. Mater. 2010, 20, 2709-2716.

(43) Hicks, J.; Behnam, A.; Ural, A. Resistivity in percolation networks of one-dimensional elements with a length distribution. Phys. Rev. E 2009, 79, 012102.

(44) Moniruzzaman, M.; Winey, K. I. Polymer Nanocomposites Containing Carbon Nanotubes. Macromolecules 2006, 39, 5194-5205.

(45) Bostanci, U.; Abak, M. K.; Aktas, O.; Dana, A. Nanoscale charging hysteresis measurement by multifrequency electrostatic force spectroscopy. Appl. Phys. Lett. 2008, 92, 093108-3.

(46) Trouwborst, M. L.; Martin, C. A.; Smit, R. H. M.; Guédon, C. M.; Baart, T. A.; van der Molen, S. J.; van Ruitenbeek, J. M. Transition Voltage Spectroscopy and the Nature of Vacuum Tunneling. Nano Lett. 2011, 11, 614-617.

(47) Huisman, E. H.; Guédon, C. M.; van Wees, B. J.; van der Molen, S. J. Interpretation of transition voltage spectroscopy. Nano Lett. 2009, 9, 3909-13. 